systemic lupus erythematosus (SLE) largely depends on migration of pulmonary artery smooth muscle cells (PASMCs). In this study, we tested whether IgG from SLE with PAH have stimulatory effects on PASMC migration.

Methods Sera from 6 SLE patients, including 1 with PAH, and 7 healthy subjects were collected, and IgG was purified using protein A or protein G. PASMC migration was examined by a Boyden chamber method. Lamellipodia formation and antibody binding sites in the cells were examined by immunocytochemistry. Identification of anti-enolase 1 antibodies was performed by immunoprecipitation, western blotting, mass spectrometry, and ELISA.

Results IgG from SLE with PAH significantly increased migration of PASMCs than those without PAH in a concentration dependent manner $(p<0.001)$. After incubation with $\operatorname{IgG}$, the number of cells with lamellipodia, which represents rearrangement of the cytoskeleton necessary to migration, was 1.4-fold higher in SLE with PAH than those without PAH $(p<0.01)$. In immunocytochemistry, IgG from SLE with PAH were colocalized with b-tubulin in the cytoplasm of PASMCs, and western blotting showed that the antibodies bound to $\mathrm{a} \sim 50 \mathrm{kD}$ protein in the lysates, which was subsequently identified as enolase1 reported to be involved in cell migration. Furthermore, the titer of IgG anti- enolase1 antibodies was 1.5 -fold higher in SLE patients with PAH than those without PAH.

Conclusions IgG from a patient with SLE accompanied by $\mathrm{PAH}$ promoted a migration of PASMCs, which is possibly ascribed to autoantibodies to enolase1.

\section{A CHALLENGE IN THE MANAGEMENT OF LUPUS NEPHRITIS WITH ACUTE KIDNEY INJURY, HEART FAILURE ON HEMODIALYSIS AND ORAL WARFARIN THERAPY: A CASE REPORT}

M Khalid. Mansoura faculty of medicine- Mansoura University, Nephrology, Mansoura, Egypt

\subsection{6/lupus-2017-000215.169}

Background and aims Systemic lupus represents a major autoimmiune disorder that leads to different major injuries to different organs of the body. Lupus nephritis can present with different disorders like nephrotic syndrome, acute kidney injury and rapidly progressive glomerulonephritis. Wise and tailored management of these cases is a must for nephrologists in order to gain the complete remission with the least side effects. If lupus nephritis is associated with other organs problems like heart failure, prosthetic valves and oral warfarin therapy, it needs more wise management. We clarify the tailored management of lupus nephritis induced nephrotic syndrome and acute kidney injury meanwhile the patient on oral warfarin therapy for prosthetic valve replacement and subsequent heart failue.

Methods We report a case of heart failure with mitral and aortic valves replacement on oral warfarin therapy. She had nephrotic syndrome, AKI due to lupus nephritis. The patient recieved high dose of oral steroid and maintained on hemodialysis for 2 months with full recovery of the AKI and partial recovery of the nephrotic syndrome.

Results The patient recieved oral high dose steroid only. Partial remession of the nephrotic syndrome occured with full recovery of the acute kidney injury and she was maintained on once per week ultrafiltration session with improvement of the ejection fraction of the heart.

Conclusions Lupus nephritis can present with complex situations. Individualization and tailoring the management for every patient in order to gain complete remission represents a challenge for nephrologists.

\section{THE CLINICAL AND LABORATORY FEATURES OF PATIENTS WITH SYSTEMIC LUPUS ERYTHEMATOSUS IN KYRGYZSTAN}

${ }^{1} G$ Koilubaeva, ${ }^{1} G$ Koilubaeva*, ${ }^{2} T$ Reshetnyak, ${ }^{2} E$ Aseeva, ${ }^{2} S$ Soloviev, ${ }^{2} E$ Nasonov, ${ }^{1}$ A Djumagulova, ${ }^{1} \mathrm{~V}$ Eralieva, ${ }^{1} \mathrm{E}$ Karimova. ${ }^{1}$ National Centre of Cardiology and Internal Medicine, Rheumatology, Bishkek, Kyrgyzstan; ${ }^{2}$ Institute of Rheumatology at Russian Academy of Medical Science, Rheumatology, Moscow, Russia

\subsection{6/lupus-2017-000215.170}

Background and aims There are no data regarding the real-life picture of SLE in Kyrgyzstan.

Methods In a prospective observation included 325 patients with SLE, who were treated in NCCIM (2012 - 2016). Majority of these patients (301) were young women (median $27[25 ; 41])$, primarily Kyrgyz (284), with disease duration of 3 (median-3,0 [0.7;8.0]) years. Assessed SLEDAI 2K and SDI.

Results They were mostly patients with acute variant of SLE129 (39.7\%), 127 (39.08\%) were with high and $86(26.46 \%)$ with very high activity. Most of the patients - 283 (87.1\%) were registered with immunological activity. In most cases of the desease were: skin lesions $(97.23 \%)$, serous membrane lesions (65.54\%) and kidney lesions (59.38\%). The neurological symptoms was noted in 120 patients (36.92\%): 99 of 120 patients $(82.5 \%)$ had a significant CNS lesions; 32 patients of $99(32.3 \%)$ had neuropsychiatric disorders, 27 of these patients had visual and auditory hallucinatory syndrome. Respiratory disorders occurred in $60(18.46 \%)$ patients. The vast majority of these patients were with pulmonary arterial hypertension (46.66\%). Acute lupus pneumonitis was detected in 23 (38.33\%) patients. At the onset of the study, SDI was identified in 65 patients $(20 \%)$. These were mainly patients with irreversible changes in the kidney $(30,8 \%)$, associated with taking GC (27.7\%).

Conclusions Acute onset of the disease (39.7\%) was noted in most Kyrgyz patients. $97.23 \%$ of those ones with primary skin lesions, $65.54 \%$ with serous membrane lesions and 59.38\% with kidney lesions, $39.92 \%$ patients had various neurological symptoms, $32.3 \%$ of these patients had serious neuropsychiatric disorders.

\section{COMORBIDITIES IN PATIENTS WITH SYSTEMIC LUPUS ERYTHEMATOSUS PRIOR TO AND FOLLOWING DIAGNOSIS: CASE-CONTROL STUDY}

${ }^{1}$ SF Luo, CF Kuo². 'Chang Gung Memorial Hospital, Rheumatology- Allergy and Immunology, Taoyuang, Taiwan R.O.C; ${ }^{2}$ CGMH, Rheumatology- Allergy and Immunology, Taoyuang, Taiwan R.O.C

\subsection{6/lupus-2017-000215.171}

Background and aims Systemic lupus erythematosus (SLE) and may associate with several categories of comorbidities. We conducted this population-based study to examine the risk of a comprehensive range of comorbidities in patients with SLE compared with matched controls. 
Methods The UK Clinical Practice Research Data-link (CPRD) was used to identify 1605 incident cases of SLE from 1997 to 2005 and matched $1: 4$ to 6284 controls by birth year, gender, general practice and year of first continuous registration. Odds ratios (ORs) of comorbidities at diagnosis and hazards ratios (HRs) after diagnosis of SLEs were estimated adjusting for age, sex, diagnosis year, body mass index, smoking and alcohol consumption.

Results SLE was associated with a higher risk for pre-existing comorbidities, with adjusted ORs $(95 \%$ confidence interval [CI]) of 2.25 (1.97-2.56), $3.37(2.49-4.57)$ and 3.54 (1.896.63 ) for the Charlson index of $1-2,3-4$ and $\geq 5$, respectively. SLE was associated with an adjusted HR (95\% CI) of 1.30 (95\% CI, 1.13-1.49) for developing new comorbidity after the SLE diagnosis. SLE was associated with a greater risk for cancer, cardiovascular, renal, liver, rheumatological and neurological diseases as well as hypothyroidism, psychosis and anaemia. The development of comorbidities was most frequent in the first two years of SLE diagnosis. Patients with SLE also had high risk of death compared with the control group, with a HR of 1.91 (95\% CI, 1.62-2.26).

Conclusions SLE patients had a burden of pre-existing comorbidities at diagnosis and the risk of development of multiple comorbidities were higher after the diagnosis compared to matched controls.

\section{THE PHARMACOTHERAPEUTIC PATTERN OF LUPUS NEPHRITIS PATIENTS AND ITS EFFECT ON THE HOSPITALISATION COST IN CHINA}

T. Li. Renji Hospital- Shanghai JiaoTong University School of Medicine, Department of Rheumatology, Shanghai, China

\subsection{6/lupus-2017-000215.172}

Background and aims To investigate the pharmacotherapeutic pattern of patients with lupus nephritis in China and its impact on the hospitalisation cost.

Methods Data were identified by the primary diagnosis of lupus nephritis from the electronic medical record system for retrospective analysis. All treatment drugs were divided into rheumatic drugs and non-rheumatic drugs for study. The hospitalisation expenses, drug utilisation rate, and its impact on the total cost were analysed.

Results 305 patients with LN between January 2014 and December 2015 were included in this study. The average hospitalisation cost was $\$ 2109.26$, including medical service fees, nursing fees, diagnosis fees, etc. Among them, drug cost was accounted for the high proportion (\$1041.41, 49.37\%), of which the non-rheumatic drugs were accounted for the major proportion (\$892.16,87.70\%). In non-rheumatic drugs, alimentary system drug had the high utilisation rate $(\$ 147.74$, 95.03\%); antimicrobial drugs had the most per person cost $(\$ 581.76,55.30 \%)$. According to the result of principal component analysis, the first principal component, which contains antimicrobial drugs and the alimentary system drugs, accounted for $77.3 \%$ cumulative variance contribution rate. Therefore, the cost of the first principal component had a great impact on the total cost. Based on result of the association analysis, the prescription of $\$ 1$ GCs would produce $\$ 2.76$ alimentary system drugs and $\$ 2.76$ anti-osteoporosis drugs to prevent adverse reactions of GCs.
Conclusions Decrease in the cost of antibiotics and alimentary system drugs could significantly reduce the patient's hospitalisation expenses. To save the disease burden, the cost of drugs for the prevention of GC adverse reactions also should be properly managed.

\section{ELDERLY SLE PATIENTS WITH PREVIOUS SKIN HERPES INFECTION AND HIGHER DOSE OF STEROID ENHANCED THE RISK OF SEVERE HERPES SIMPLEX INFECTION: A NATIONWIDE STUDY}

${ }^{1,2} \mathrm{TH}$ Li*, ${ }^{3}$ YS Chang, ${ }^{4} \mathrm{CC}$ Lai, ${ }^{4} \mathrm{CY}$ Tsai. ${ }^{1}$ Taichung Venterans general hospital - Chiayi branch, Division of Allergy- Immunology- and Rheumatology- Department of Medicine, Chiayi City, Taiwan R.O.C; ${ }^{2}$ National Yang-Ming University, Institute of Clinical Medicine, Taipei, Taiwan R.O.C; ${ }^{3}$ Shuang-Ho Hospital- Taipei Medical University, Division of AllergyImmunology- and Rheumatology-Department of Internal Medicine, New Taipei City, Taiwan R.O.C; ${ }^{4}$ Taipei Veterans General Hospital, Division of Allergy- Immunology- and Rheumatology-Department of Medicine, Taipei City, Taiwan R.O.C

\subsection{6/lupus-2017-000215.173}

Background and aims Systemic lupus erythematosus (SLE) patients are susceptible to herpes simplex virus (HSV) infection, which is occasional but often leading to overwhelming disease such as encephalitis and keratitis. However, only few attempts have been made at the associated incidence and risk factors.

Methods We enrolled SLE patients from the Taiwanese National Health Insurance research database between 1997 and 2012. We compared the incidence rate (IR) of severe HSV infection, including viral septicemia, meningoencephalitis, ocular infection, visceral infection and those with complications after infection, with those of non-SLE cohort. We also evaluated the risk factors of severe HSV infection by means of Cox multivariable proportional hazards model.

Results A total of 122520 subjects (24 504 SLE patients and 98016 age- and gender-matched subjects as control group) were analysed and revealed a significantly higher IR of severe HSV infection in SLE (Incidence rate ratio=3.52, $\mathrm{p}<0.001$ ). Previous skin infection $(\mathrm{HR}=2.17, \mathrm{p}=0.047)$, intravenous steroid pulse therapy $(\mathrm{HR}=4.48, \mathrm{p}<0.001)$, oral daily steroid dose over $7.5 \mathrm{mg}$ prednisolone or equivalent $(\mathrm{HR}=1.60$, $\mathrm{p}=0.010$ ) were independent risk factors for severe HSV infection in SLE patients, while age $\leq 18(\mathrm{HR}=0.47, \mathrm{p}=0.021)$ was a protective factor.

Conclusions A higher risk of severe HSV infection was observed in SLE patients The risk factors for severe HSV infection were age over 18, previous skin infection, intravenous steroid pulse therapy and an oral daily steroid dose over $7.5 \mathrm{mg}$.

\section{THE RELEVANCE OF VASCULAR EVALUATION IN SYSTEMIC LUPUS ERYTHEMATOSUS. RESULTS FROM AN INCEPTION COHORT}

${ }^{1} \mathrm{R}$ Lizola*${ }^{1} \mathrm{CA}$ Hinojosa, ${ }^{1} \mathrm{H}$ Laparra, ${ }^{1} \mathrm{JE}$ Anaya-Ayala, ${ }^{2} \mathrm{LA}$ Mendoza, ${ }^{2} \mathrm{M}$ Ocampo, 2J Romero-Díaz. "Instituto Nacional de Ciencias Médicas y Nutrición "Salvador Zubirán", Vascular Surgery, Mexico city, Mexico; ${ }^{2}$ Instituto Nacional de Ciencias Médicas y Nutrición "Salvador Zubirán", Rheumatology, Mexico City, Mexico

10.1136/lupus-2017-000215.174

Background and aims Systemic lupus erythematosus (SLE), as a chronic inflammatory disease, has been associated with 